\title{
Robotic Assembly of Truss Structures for Space Systems and Future Research Plans'
}

\author{
William Doggett \\ NASA Langley Research Center \\ MS: 152D \\ Hampton, Va. 23662 \\ w.r.doggett@larc.nasa.gov
}

\begin{abstract}
Many initiatives under study by both the space science and earth science communities require large space systems, i.e. with apertures $>15 \mathrm{~m}$ or dimensions $>20 \mathrm{~m}$. This paper reviews the effort in NASA Langley Research Center's Automated Structural Assembly Laboratory which laid the foundations for robotic construction of these systems. In the Automated Structural Assembly Laboratory reliable autonomous assembly and disassembly of an 8 meter planar structure composed of 102 truss elements covered by 12 panels was demonstrated. The paper reviews the hardware and software design philosophy which led to reliable operation during weeks of near continuous testing. Special attention is given to highlight the features enhancing assembly reliability.
\end{abstract}

\section{TABLE OF CONTENTS}

1. INTRODUCTION

2. LITERATURE SURVEY AND OTHER ACTIVITIES

3. OVERVIEW

4. EXAMPLE ASSEMBLY

5. SUMMARY OF CRITICAL FEATURES

6. FUTURE WORK

7. Closing Comments

\section{INTRODUCTION}

Many initiatives under study by both the space science and earth science communities require space systems with large apertures, i.e. $>10 \mathrm{~m}$. These initiatives include the Terrestrial Planet Imager, Tropical Rain Forest Measurement system and Space Solar Power. Several techniques are available or under development to support construction of these large space systems including inflation of gossamer structures or elements, thin film gossamer membranes for reflectors, advanced deployable systems, and on-orbit assembly. Gossamer technology development is in its infancy, however, if successful it has the capability of producing deployed areal densities of less than $5 \mathrm{~kg} / \mathrm{m}^{2}$. While gossamers are a promising area, there are many unsolved issues including deployment strategy, shape control and debris/meteor impact resistance that have yet to be solved. Deployable techniques are the conventional technique for fielding space systems, but they suffer from increased complexity (lower reliability) as size increases and are ultimately limited by the launch vehicle capabilities.[1] On-orbit assembly of space structures introduces many advantages. These include inherent serviceability, inherent expandability, launch packing efficiency, ability to launch small units independently, incremental system upgrades, structural efficiency (areal densities of $15 \mathrm{~kg} / \mathrm{m}^{2}$ for conventional hardware), essentially no increase in complexity with size, and the ability to build very large structures (i.e. $>100 \mathrm{~m}$ ). The disadvantage of on-orbit assembly lies in the support system required to perform the assembly. However, the system is inherently reusable, i.e. can be applied to the construction of several components and does not increase in complexity with increasing structural size. In addition, the support system directly supports operational maintenance and repair. Once a structural concept has been defined, the actual assembly is a well-defined repetitive operation, ideally suited to robotic techniques.

In the early 90's researchers at NASA Langley Research Center realized the potential for automated assembly of space structures and began the development of a robotic system to assemble truss structures with equal length members.[2] Truss assembly was selected because trusses form the primary support structure in many missions including aerobrakes, telescopes, and solar array fields.[3] The robotic work culminated in the repeated autonomous assembly and disassembly of an $8 \mathrm{~m}$ diameter structure composed of 102 truss members covered with 12 panels shown in Figure 1. It is important to note that the assembly and disassembly occurred in an unstructured lighting environment.

This paper begins by reviewing the significant activities in robotic space construction. Following the review, the remainder of the paper focuses on the work at NASA Langley Research Center, beginning with an overview of the Automated Structure Assembly Laboratory (ASAL) highlighting robot friendly features in the truss hardware. Then an example truss assembly is used to illustrate the lessons learned during repeated hardware tests.[4] The paper closes by introducing plans to extend the effort to the assembly of a doubly curved space radiometer structure formed from EVA/robotic compatible hardware shown in Figure 14. The objective of this on-going effort is to 


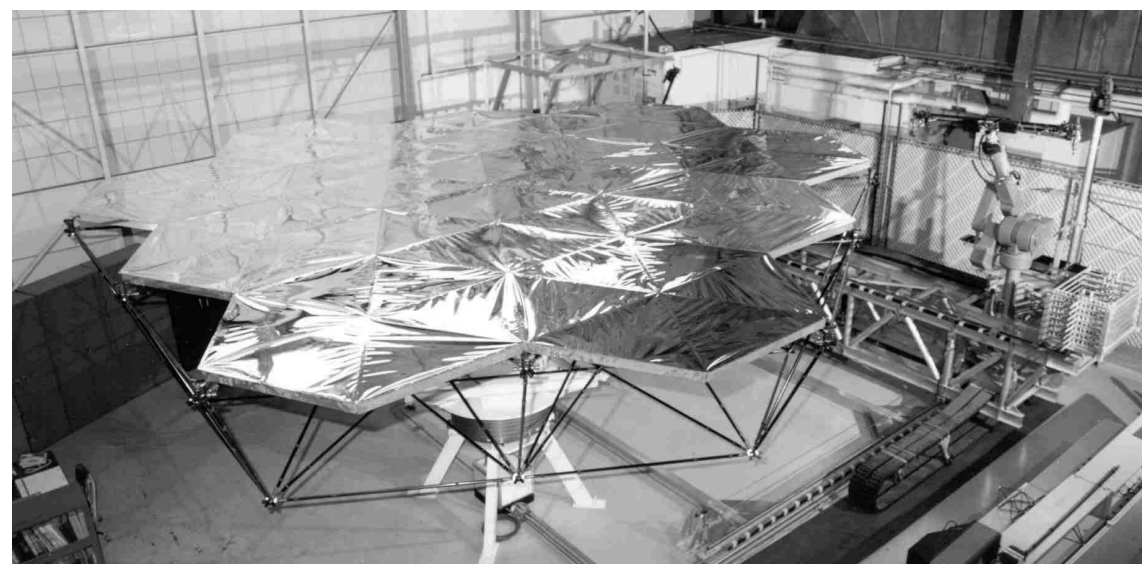

Figure 1. Robotically Assembled Tetrahedral Truss Structure with Panels

that enabled higher density packing.[10] Both joints had merit, but require tools for EVA use.

There has also been extensive research in telerobotics, i.e. robotic systems under the direct control of a human operator. The Remote Manipulator System on board the US Space Shuttle, the Space Station Remote Manipulator System, and the Japanese Remote Manipulator System are three examples of telerobotic systems.[11, 12]

Following the successful Assembly Concept for Construction of Erectable generalize and validate robotic assembly techniques for a complete space system so that they may be incorporated into a flight program. Further, this effort in conjunction with the extensive EVA database on truss assembly enables a comparison of robotic and EVA assembly "head to head" for a representative space structure.[5]

\section{LITERATURE SURVEY AND OTHER ACTIVITIES}

In parallel to the robotic activity discussed here, NASA Langley Research Center has conducted complementary research in structures for on-orbit assembly and EVA assembly techniques. These efforts are covered by a companion paper in this same session titled "A History of Astronaut Construction of Large Space Structures at NASA Langley Research Center" by Ms. Judith Watson.[6]

In the robotic construction arena, several significant activities were under way to research alternative techniques for joining the structural members. Jones and Thomas of Marshal Space Flight Center investigated on-orbit welding of structures as an alternative to joint assembly.[7] These systems provided a light structural connection, but suffer from high power requirements, lack of serviceability, requirement for on-orbit inspection, and out gassing contamination.[8] Dick et. al. investigated modifications to the Langley EVA joint design to improve the ability of robots to use the joint.[9] Brewer investigated joint designs

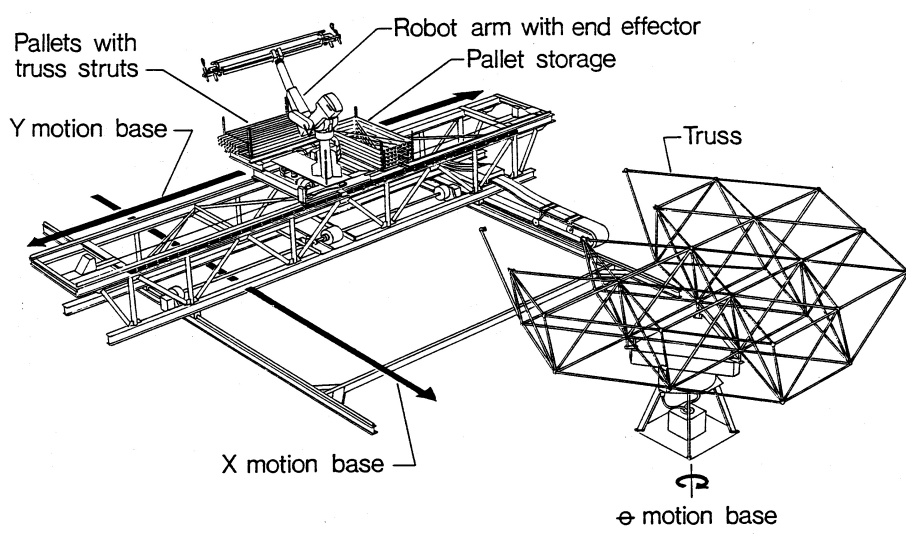

Figure 2. Automated Structures Assembly Laboratory

Space Structure (ACCESS) flight experiment, researchers at Oak Ridge National Laboratory demonstrated the feasibility of telerobotic construction using similar hardware.[13,14] Though not discussed in this paper, a telerobotic control station, such as that suggested by Akin et. al., used in conjunction with the system discussed in this paper would significantly enhance its operational viability by enabling unplanned inspection and repair operations to be performed by ground operators.[15] The validity of ground control was verified by Hirzinger et. al. who controlled ROTEX on board the U.S. Space Shuttle from a ground station and they also participated in the ground control of the Japanese ETS VII space free flyer.[16,17,18,19]

In addition to the efforts in telerobotic systems, several researchers are working on fully autonomous systems. For example, Whittaker et. al. are developing generalized autonomous robotic systems for space assembly, inspection, and maintenance. [20,21] These systems will lead to routine unsupervised activities, such as inspection and maintenance.

\section{OVERVIEW}

This section provides an overview of the physical hardware used in the ASAL. Special attention is made to highlight a focus on system robustness, through sensor verification and passive alignment features of the hardware, which is critical to the reliable operation of the automated system.

The ASAL, depicted in Figures 1 and 2, includes three motion bases, an industrial robot, two special purpose endeffectors, and several surveillance cameras. The truss structure is assembled in rings on a rotary motion base, visible in the center of Figure 1 and the right of Figure 2, that presents the unfinished portion of the structure to the robot. The robot, on the right of Figure 1 rides on two linear motion bases that position it for strut or panel installation. The robot is a commercially available six-degree-of-freedom manipulator arm with a 60 -inch reach and a $30-1 \mathrm{~b}$ payload capacity. No custom modifications of the robot were required to support assembly operations. Truss members are stored in pallets behind the robot (Figure 2). After all truss 


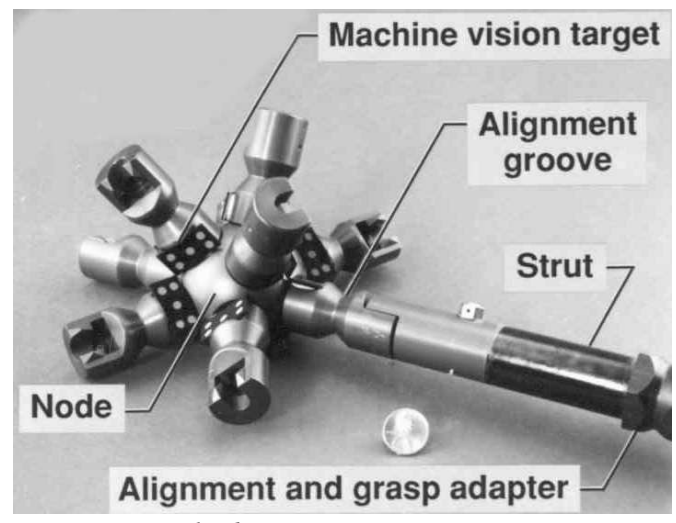

Locked Joint

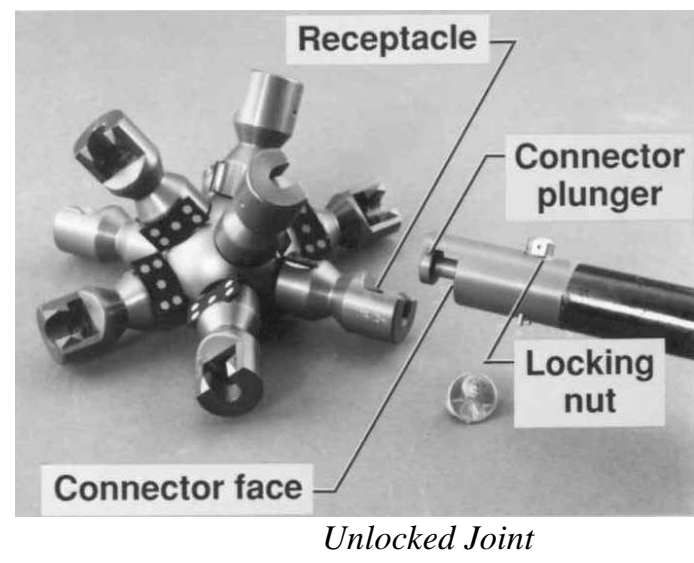

Unlocked Joint

Figure 3. Truss Components

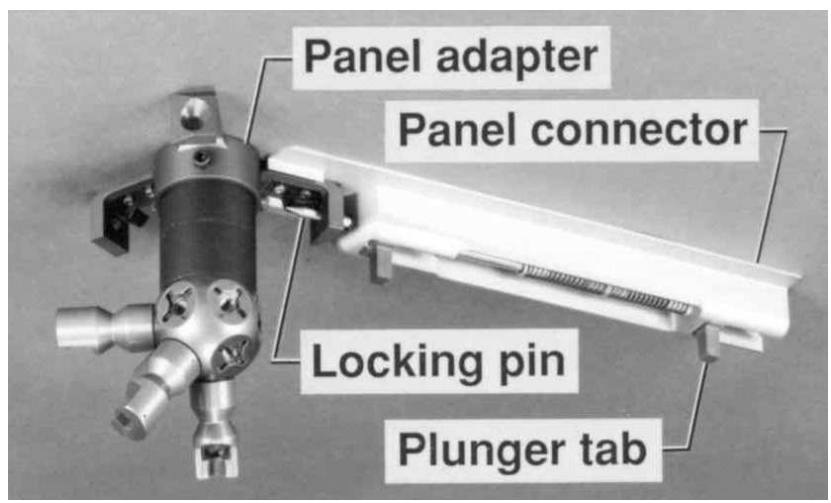

Figure 4. Panel Components

members from a pallet have been installed, the pallet is moved to a pallet storage location at the side of the robot.

Figure 3 shows the hardware components used to assemble the truss structure. The nodes are used to position the receptacles to form a specific structural geometry. The receptacles form half of the joint and include an alignment groove that is grasped by the end-effector receptacle fingers to fix the end-effector position during strut installation. The other half of the joint is affixed to the strut and includes a locking nut that is rotated by a nut driver on the endeffector, visible in Figure 6, to secure the joint by drawing the connector plunger into the strut half of the joint. The alignment and grasp adapter is shaped to maintain the precise location and orientation of the strut while being transported by the end-effector. Machine vision targets are located at the base of the receptacles. These targets are used to estimate the location of the receptacle during the final approach to the structure.[22] Panels are attached using the hardware shown in Figure 4. Panel adapters are affixed to the nodes forming the top surface of the structure. Panel connectors are affixed to the underside of the panels. The panel connectors secure the panels in the structure, storage canister, or on the end-effector for transport to the structure. The end-effector engages the plunger tab, sliding the plunger to the right in the figure to withdrawal the locking pin from the panel adapter releasing the panel while simultaneously extending the other end of the pin into a mating hole in the end-effector, thus securing it for transport. The specially designed endeffector shown in Figure 6 was designed to insert and secure truss members forming the structure. The endeffector

mechanically

symmetric about its center. At the lower left of the figure the mechanisms inset shows the endeffector holding a strut for transport via the strut holder. Also shown are the receptacle fingers used to register the end-effector to the structure by closing on the alignment groove in the receptacle. The passive alignment provided by the oversized receptacle fingers and corresponding alignment groove in the receptacle shown in Figure 5 are key design features that significantly enhance the operational robustness of the system. Insertion of the strut occurs by extending the insertion platform and locking the joint using the nut driver. Notice that the end-effector internally reacts all insertion forces during the installation.

The insert on the lower right of the figure details the machine vision system and associated lighting used to locate the targets shown in Figure 3. Located at the center of the end-effector in Figure 6 are the support electronics. These include an on-board embedded microprocessor used to orchestrate the end-effector operations and interface electronics used to minimize the number of signals that must pass through the quick-change mechanism. The quickchange enables autonomous end-effector exchanges to occur when transitioning from truss installation to panel installation. Two end-effector exchanges are required during the construction of each ring. A critical design requirement of ASAL is that all operations must be verifiable by sensors. Because the system has been developed to support autonomous construction at remote locations, each step of the assembly process is verified by sensor feedback. For example, after the receptacle fingers have been commanded to close on a receptacle, a sensor verifies the receptacle finger closure and a second receptacle presence sensor verifies the successful capture of the receptacle. If an unexpected situation is detected, then the system pauses, notifies the operator, and awaits the operator's directions. During assembly tests composed of 3 full assemblies and disassemblies requiring more than 110 hours of continuous operation over several weeks, only 2 such unscheduled instances occurred. Once when a facility door was opened causing a reflection from a car windshield to saturate the CCD camera so that the target could not be located and once when a bird landed on a strut during a 


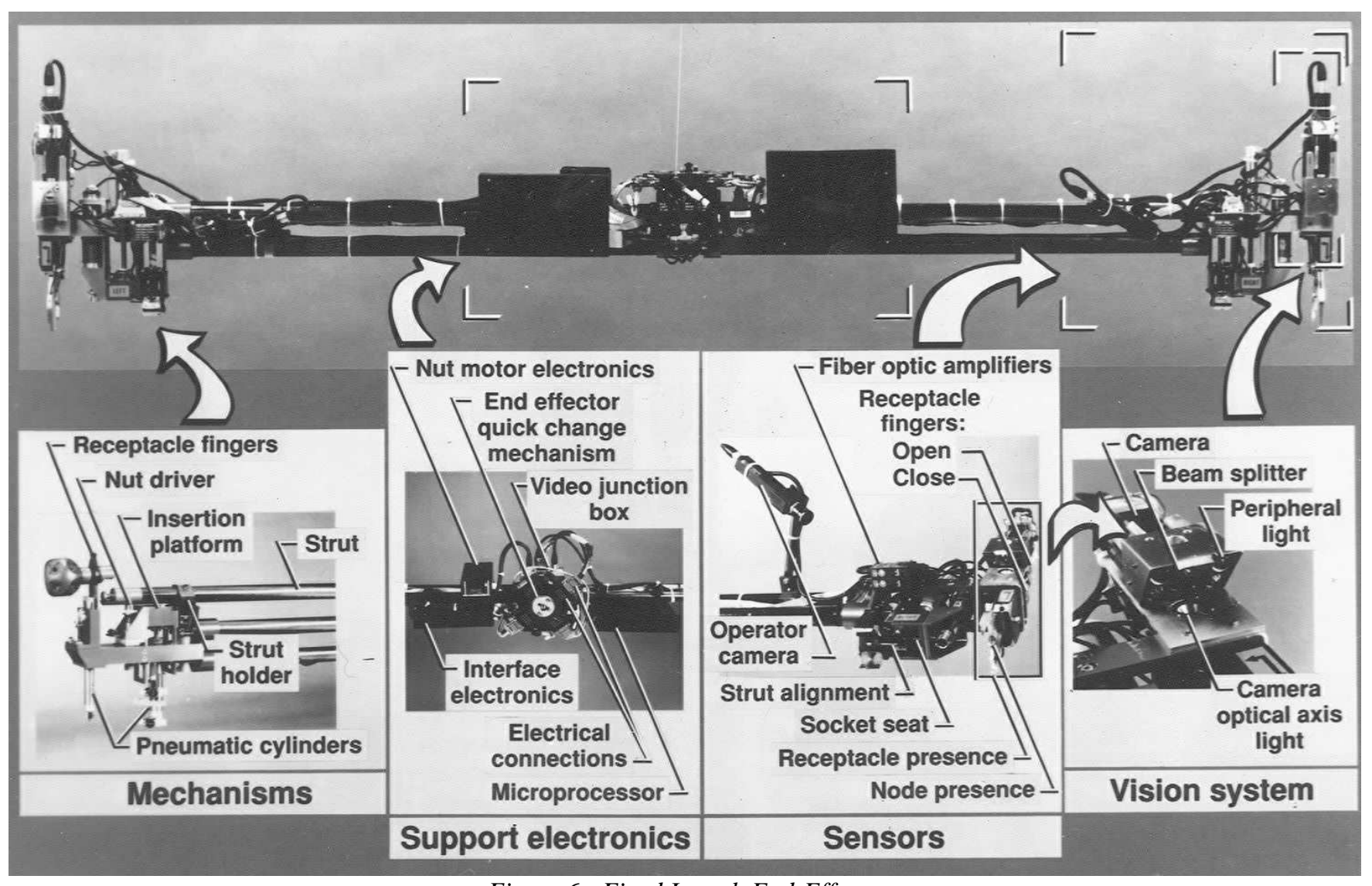

Figure 6. Fixed Length End-Effector

capture operation. In both cases the problem was eliminated and the automated system directed to reverse a few steps and then continue to resume the assembly.

The end-effector used to install or remove panels is shown in Figure 7. The end-effector shares many of the same features as the truss element end-effector, including an on board microprocessor, support electronics, and sensor verification of all functions. Similar receptacle fingers, visible at the top left of the figure are used to register the end-effector to the structure prior to panel installation. This is a key feature of the panel installation, maintaining the panel above the top structural plane until final alignment is achieved at which point the panel is smoothly lowered and locked into position.

The automated assembly system is capable o constructing both planar structures as shown ir Figure 1 as well as beam structures like tha shown in Figure 9. This paper focuses or construction of the planar geometry, the bean geometry is constructed in a similar fashion. The planar structure is composed of 102 struts and 12 panels forming a structure $8 \mathrm{~m}$ in diameter. Ful assembly required approximately 20 hours anc disassembly required approximately 18 hours.

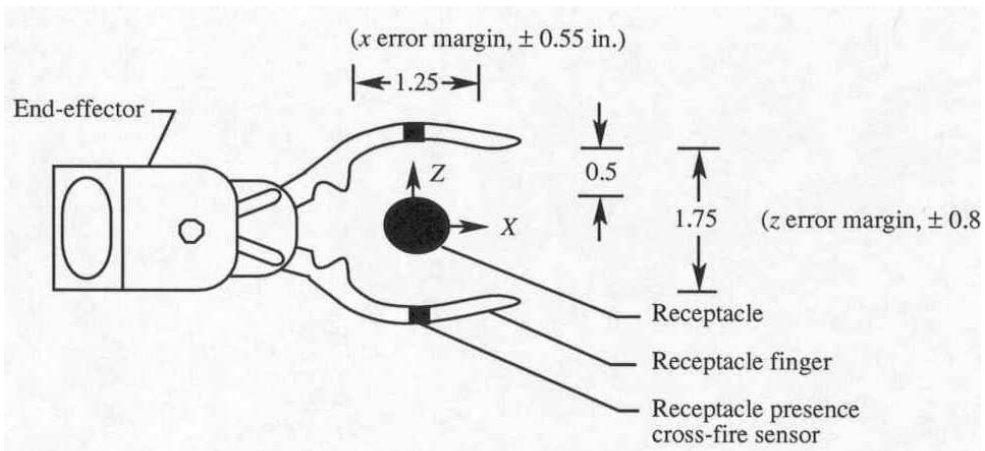

(a) Side view of receptacle and receptacle fingers.

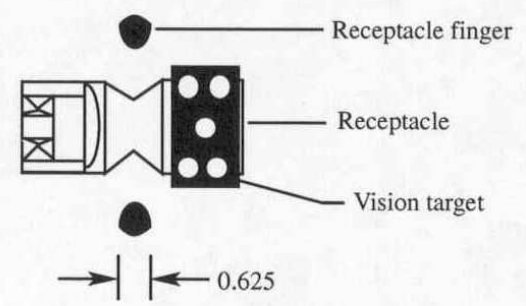

( $y$ error margin, \pm 0.25 in.)

int view of receptacle and receptacle fingers.

Figure 5. Passive Features of Receptacle and Receptacle Fingers 


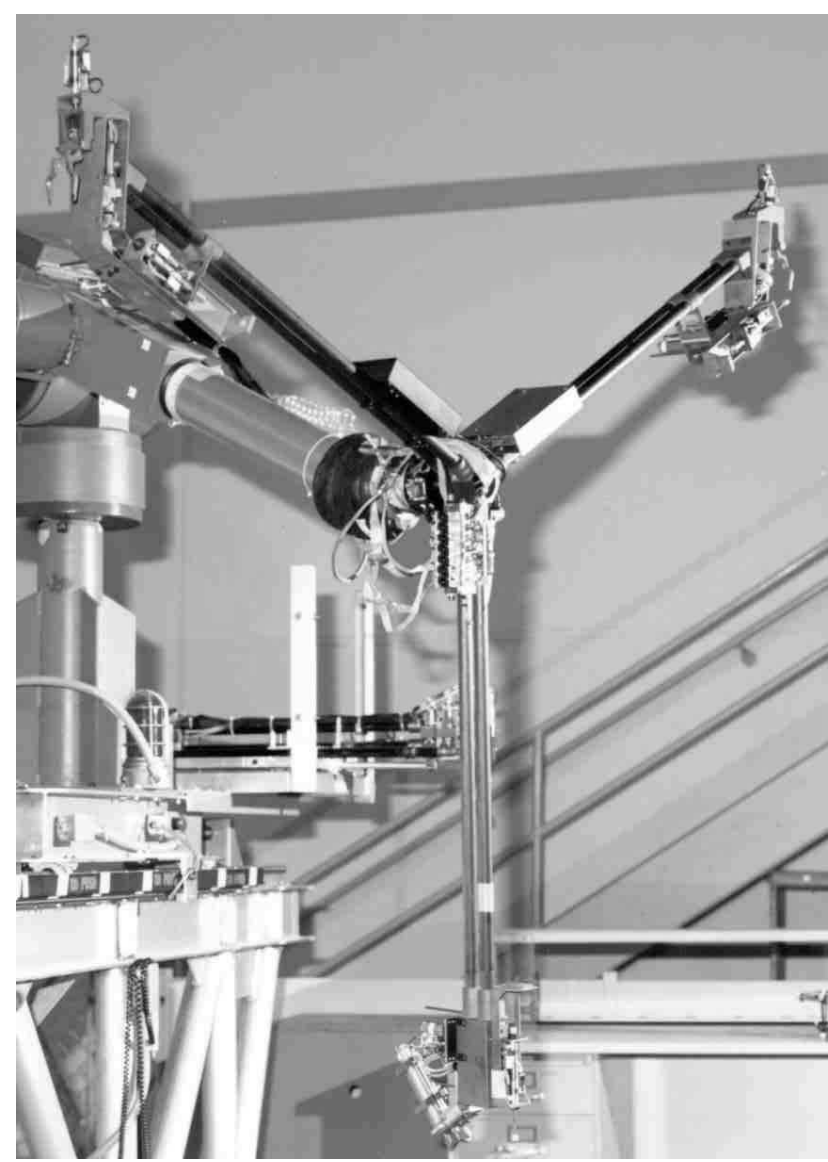

Figure 7. Panel End-Effector

\section{EXAMPLE ASSEMBLY}

This section describes the steps used to install truss elements and panels. During the description, key attributes of the system enhancing the system reliability are highlighted. These include the use of complementary sensing techniques providing overlapping coverage during different guidance phases and a hierarchical software scheme supporting system to be pauses or queries at any time during the assembly. In addition, the assembly process is designed to minimize the opportunities for collisions by maneuvering the robot in large open areas. Precise dexterous operations are performed by the specially designed endeffectors after the end-effector has been register to the hardware by gripping on alignment features.

Structural assembly alternates between assembly of rings of truss elements and rings of panels. Strut installation begins with the removal of a strut from the canister located behind the robot as shown in Figure 8. The strut is then transported to a prescribed location above the middle of the canister. Next the strut is transported to the vision approach point (VAP), approximately 12 to 16 inches from the strut's final installation position. At this point a machine vision algorithm takes over to guide the robot to the structure.[23] The path from above the canister to the structure via the VAP was calculated using an off-line path planner.[24] Key to the success of off-line path planning is the ability to plan a path to the final installation position, based solely on structural geometry. Success of the path planner provides a high degree of confidence in the ability to assemble structures too large to be completely assembled in a test facility. Inaccuracies between the planner's geometric model and the actual hardware within the facility are overcome through the use of machine vision guidance initiated at the VAP. These inaccuracies are due to slight inconsistencies in the hardware, robot positioning errors, motion base positioning errors, dynamic effects, etc. Following machine vision guidance to a point where the existing structure may be grasped by the robot, a force torque algorithm is invoked providing final precision alignment maneuvers prior to strut installation. A successful strut installation is shown in Figure 10. During the assembly, nodes are transported to the structure preattached to struts.

Once the truss elements comprising a ring of structure have been installed, the truss element end-effector is stowed on the strut pallet behind the robot and the panel end-effector is retrieved from its storage location within the panel canister. Panel installation commences with the removal of a panel from the canister (Figure 11), followed by transport to the structure (Figure 12), then alignment at the structure

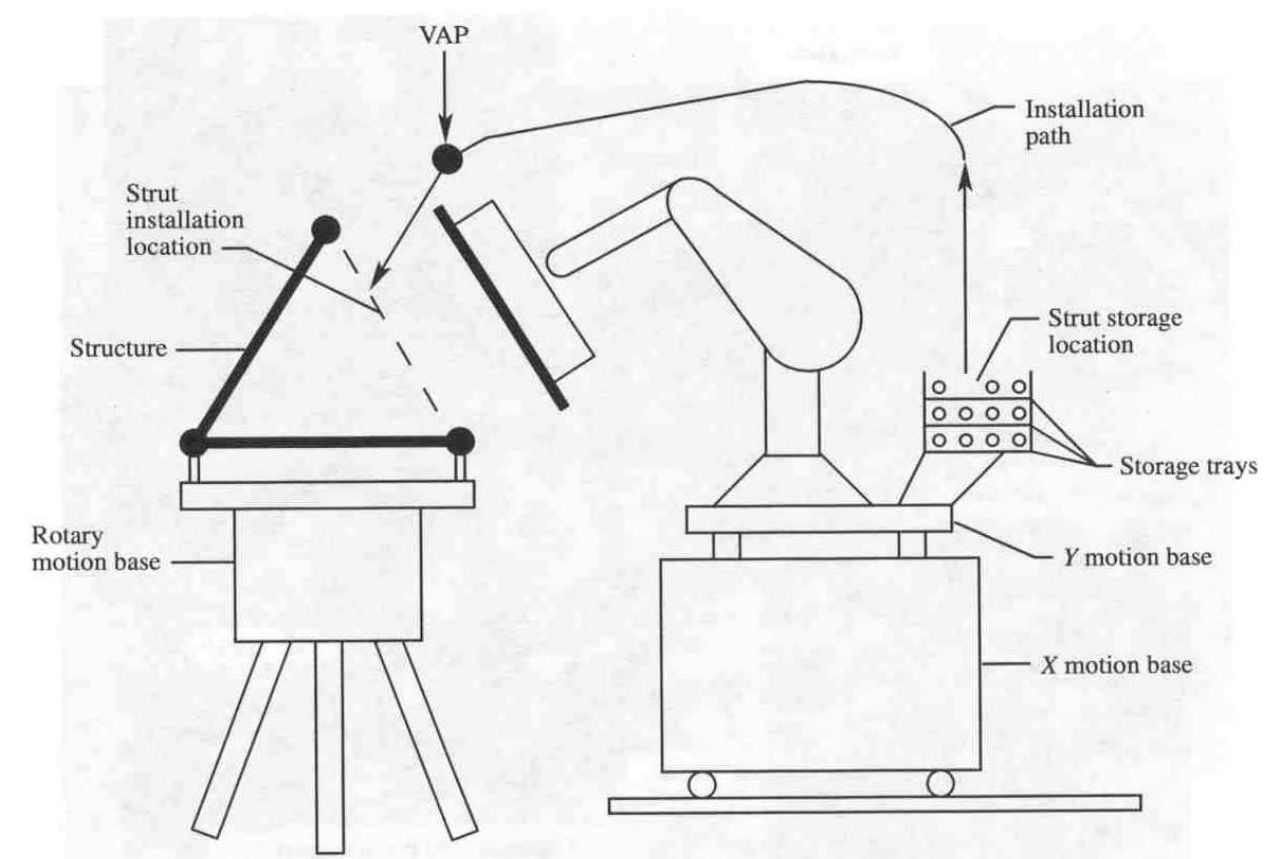

Figure 8. Generic Strut Installation 


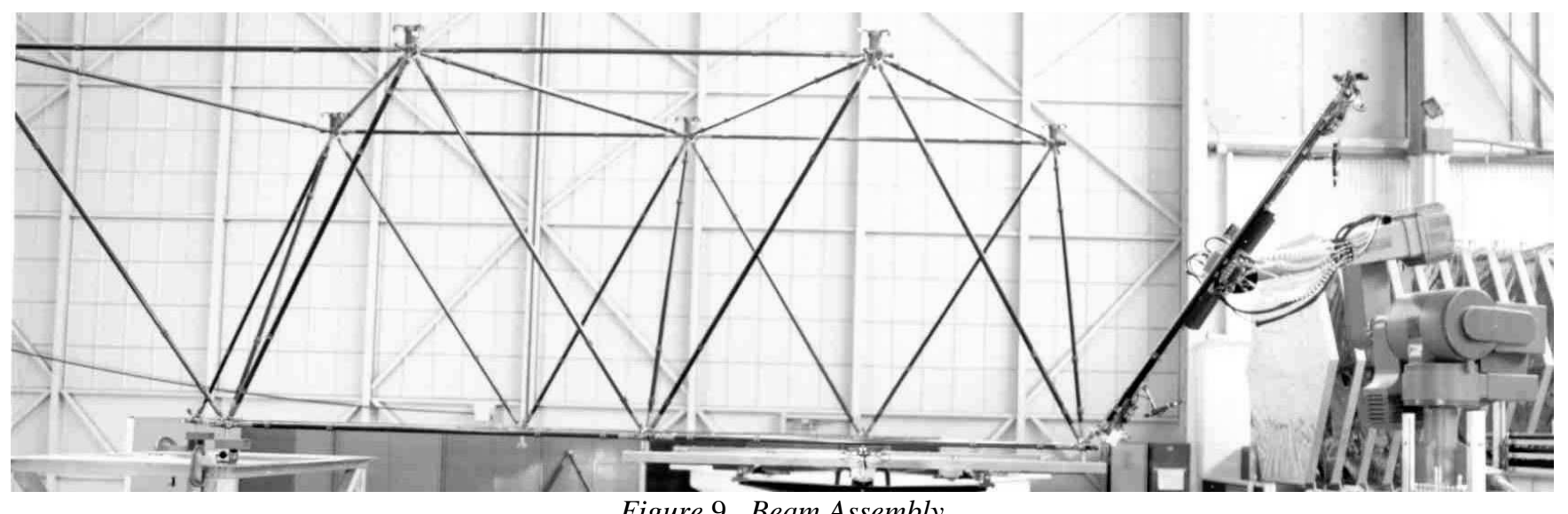

Figure 9. Beam Assembly

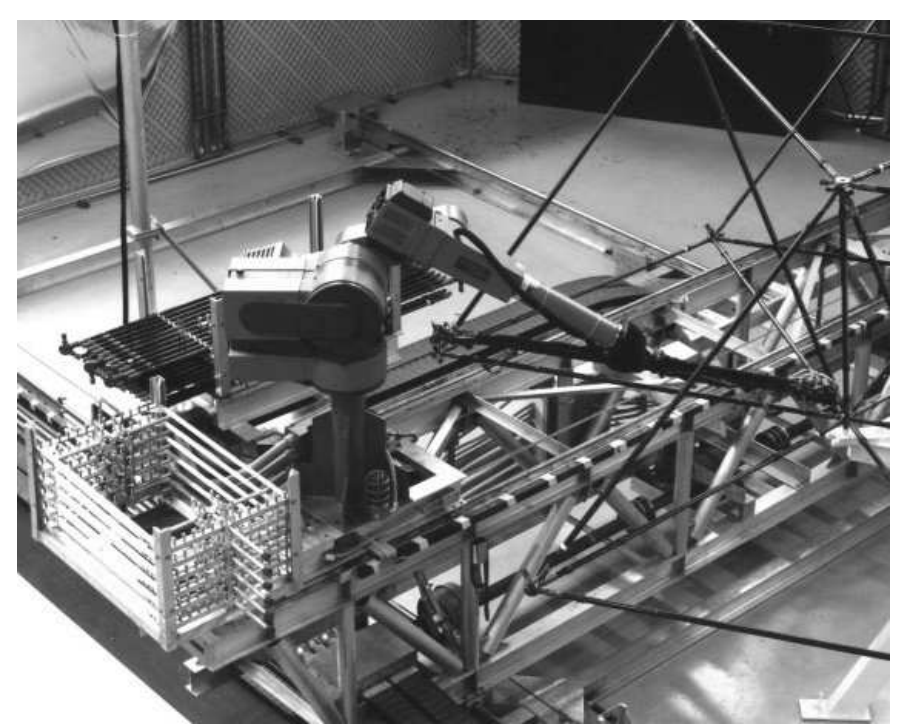

Figure 10. Robotic Strut Insertion

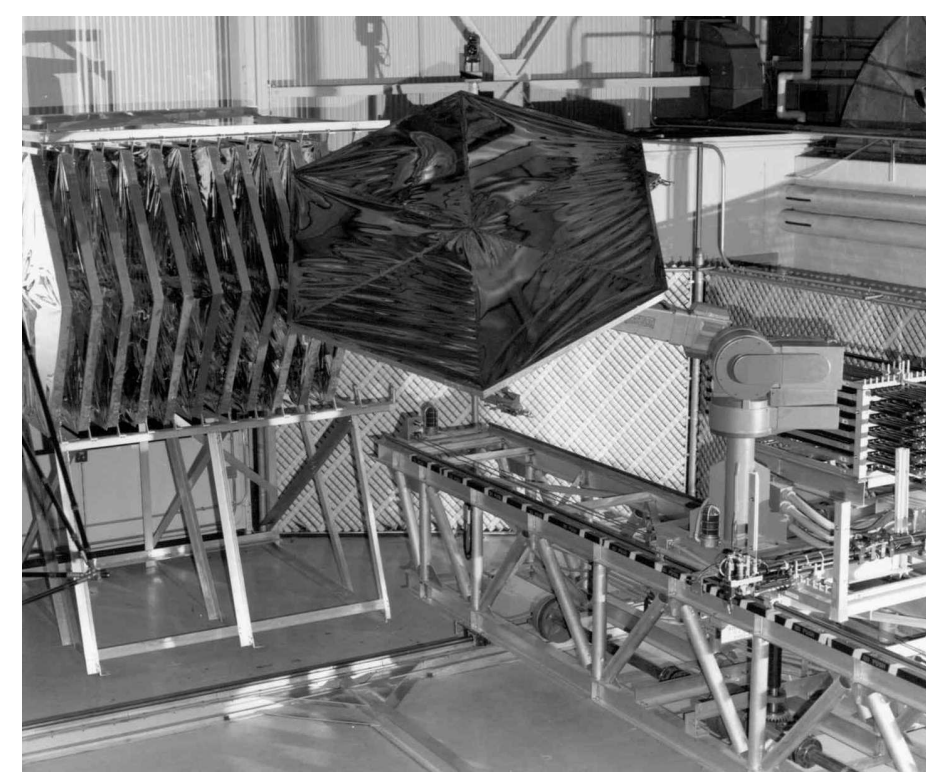

Figure 12. Panel Lifted for Approach to Structure

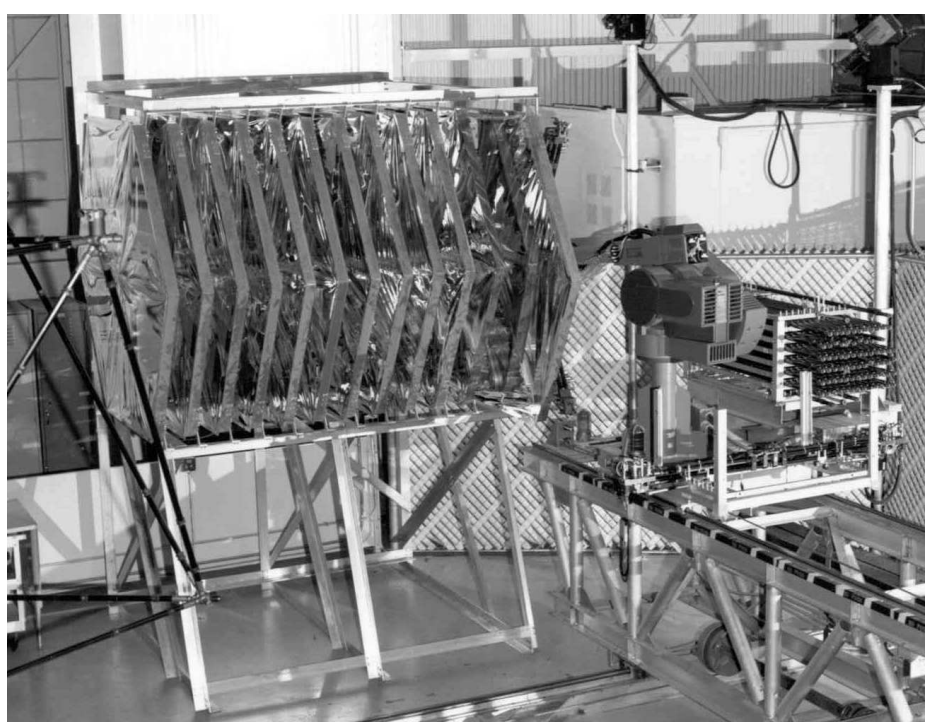

Figure 11. Panel Removal from Canister

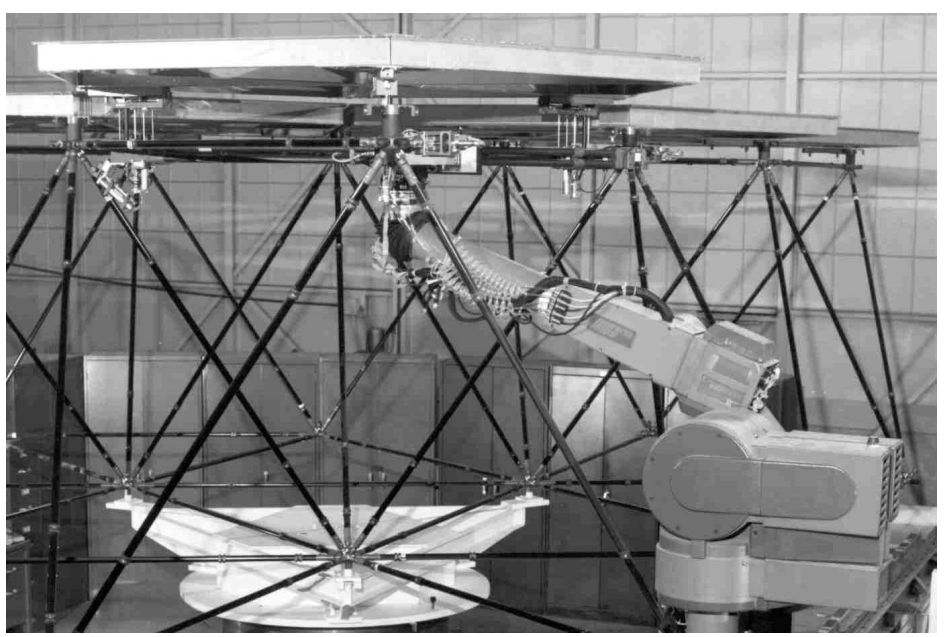

Figure 13. Panel Lowered Into Position 


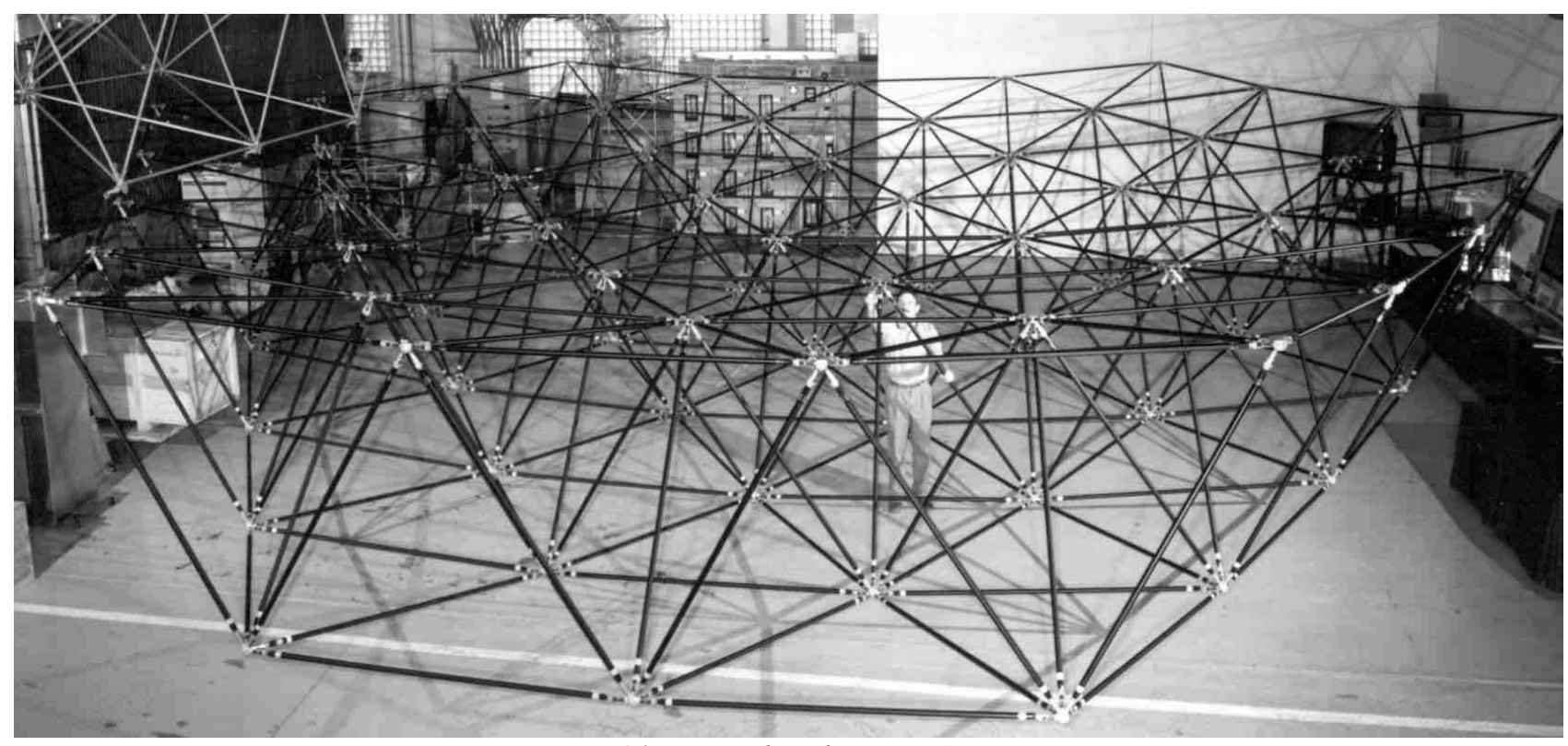

Figure 14. Curved Radiometer Structure

(Figure 13) and finally attachment to the structure. Following panel installation, the panel end-effector is rotated down and withdrawn from the structure, returning to the panel canister to begin the installation of another panel. Once all the panels in the ring are installed, the panel endeffector is stowed in the panel canister and the truss element end-effector is retrieved from the strut pallet prior to resuming truss element installation on the next ring.

Assembly is orchestrated by an executive program executing an expert system on the host computer.[25] The executive program provides the operator interface and coordinates the actions of computers dedicated to robot motion, motion base motion, end-effector control, and vision-processing. The assembly is carefully choreographed such that it may be paused or reversed at any point. Also, the operator may direct the functions of the system at any level. High level commands such as "assemble the st ructure" are provided as well as interfaces to the primitive commands for the different systems such as "move robot" or close "receptacle fingers".[26] It is also possible, through access to password protected functions, for an operator to override sensor feedback, disable checks on a particular sensor, or interactively provide sensor feedback. Further the operator may query the state of the system at anytime to assess progress or evaluate the system state following an error message. While these features may not come into play during system tests, they are critical to effect development and debug activities.

\section{SUMmARY OF CRITICAL FEATURES}

In this section the critical features of the hardware, software, and design philosophy will be summarized. These features form the foundation of the robust reliable assembly system developed and validated through years of hardware test experience.
- Always register to structure during approach and installation

- $\quad$ via machine vision

- $\quad$ via force torque and passive hardware features

- Verify all operations via sensor feedback

- Provide large capture envelopes using generous chamfering.

- React all insertion loads through the end-effector

- Provide overlapping sensor coverage during hardware approach. Enables sensor responses to be verified before moving to the next phase.

- Develop custom end-effectors maneuverable by any robot. Robots are expensive; therefore take advantage of available systems.

- Assembly process is fully reversible/pausable. Significantly reduces operator workload by enabling pauses to evaluate system performance. Naturally leads to a system supporting disassembly and repair.

- During installation struts/panels/components are kept at a safe distance until registration to the structure is complete.

- Path planning key to a reliable autonomous system enabling construction of arbitrary structures.

- Operate robot in clear regions to reduce dexterity requirements.

- $\quad$ Provide operator interfaces to all command levels.

\section{FUTURE WORK}

The ASAL demonstrated reliable robotic assembly of planar truss structures and beams. Future plans are centered around complete construction of a doubly curved radiometer designed for an off axis feed. The truss structure for this instrument is shown in Figure 14. The objectives of this effort are to generalize the assembly system so that nonplanar structures can be constructed while simultaneously demonstrating a system that represents a realistic flight 
system. To accomplish these objectives, several technical

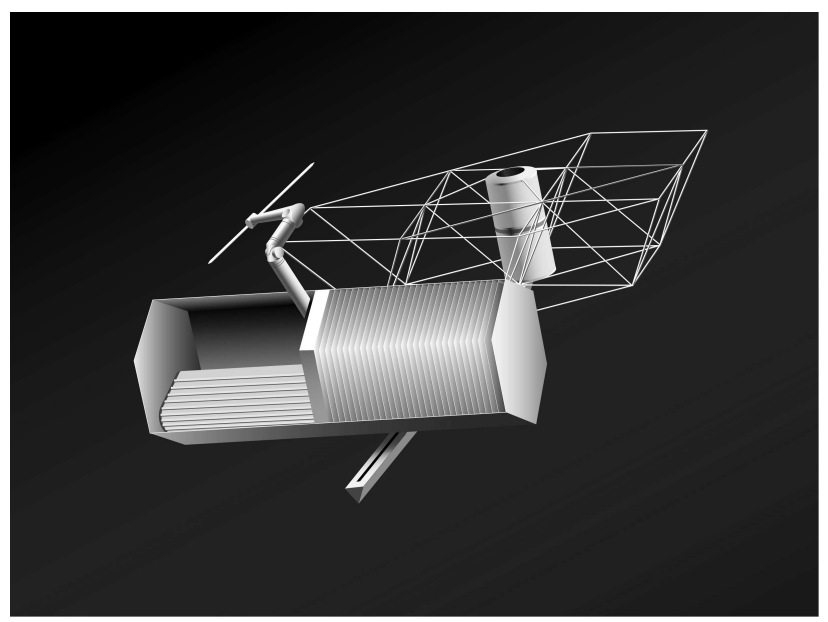

Figure 15. Conceptual Drawing of In-Space Assembly System

hurdles must be overcome. First, the y motion base of Figure 2 will be eliminated resulting in a cylindrical coordinate planning system. Second, the path planning system will be enhanced to include path planning of the motion bases. Including the motion bases complicates the planning problem because it results in the ability to install a given strut in a number of ways requiring a cost function to be developed to select the "best" path from the group of possible paths. The cost can be dependent on assembly time, energy consumption, collision avoidance metrics, etc. Third, the issue of power and control signal distribution must be addressed in parallel with the design of a robust panel attachment system. State of the art techniques, such as those proposed for the Next Generation Space Telescope, incorporate arrays of actuators in each panel to accurately position each panel, thus necessitating control and power distribution to each panel.[27] Fourth, the construction of the feed structure for secondary optics must be verified.

Figure 15 depicts an artist concept of an on-orbit assembly system. The robot is launched stored adjacent to the truss members shown on the left side of the canister and begins assembly on a rotary motion base deployed from the canister. Panels are shown in the canister on the right. Here the system is shown working from a single canister, however a straightforward extension is the aggregation of several canisters on orbit prior to transport to the assembly site to take advantage of lower cost launch services. Once the assembly is complete, the robot is powered down and stowed, available for future inspection or repair operations as required.

\section{Closing Comments}

The Automated Structures Assembly Laboratory at NASA Langley Research Center demonstrated reliable autonomous assembly of planar structures. On-orbit assembly of space structures provides many advantages including: inherent serviceability, inherent expandability, launch packing efficiency, ability to launch small units independently, incremental system upgrades, structural efficiency, essentially no increase in complexity with size, and the ability to build very large structures (i.e. > 100m). Future plans include the demonstrated assembly of a doubly curved radiometer that leads directly toward a flight experiment validating the assembly techniques on-orbit. This is the first step in an ambitious program to develop approaches for large space systems that are not limited by launch constraints.

\section{REFERENCES}

1 Lake, Mark S., et al., Research on the Problem of HighPrecision Deployment for Large-Aperture, Space-Based Science Instruments, presented at the 1998 Space Technology \& Applications International Forum, Albuquerque, New Mexico, January 25-29, 1998.

2 Will, Ralph, Marvin Rhodes, William Doggett, Catherine Herstrom, Carolyn Grantham, Cheryl Allen, Daniel Sydow, and Eric Cooper. "An Automated Assembly System for Large Space Structures." Published by Kluwer Academic Publishers as part of the book entitled Intelligent Robotic Systems for Space Exploration, 1992.

3 Doresy, John T. and Martin M. Mikulas, Jr. Preliminary Design of a Large Tetrahedral Truss/Hexagonal Panel Aerobrake Structural System, $31^{\text {st }}$ Structures, Structural Dynamics and Materials Conference, Long Beach Ca., 1990. AiAA Paper No. 90-1050.

4 Rhodes, Marvin D.; Will, Ralph W.; and Coung Quach. Baseline tests of an Autonomous Telerobotic System for Assembly of Space Truss Structures, NASA Technical Paper 3448 .

5 Lake, Mark S.; Walter L. Heard, Jr., Judith J. Watson and Timothy J. Collins, Evaluation of Hardware and Procedures for Astronaut Assembly and Repair of Large Precision Reflectors, NASA/TP-2000-210317, August 2000.

6 Watson, Judith; Collins, Timothy and H. G. Bush. "A History of Astronaut Construction of Large Space Structures at NASA Langley Research Center", 2002 IEEE Aerospace Conference, Big Sky Montana.

7 Rule, William K. and Frank P. Thomas. "Welded Joints for Robotic, On-Orbit Assembly of Space Structures", Journal of Aerospace Engineering, Vol. 7 num. 2, April 1994, pp 209-24.

8 Jones, Clide S. and Frank Thomas. 'Robotic Assembly of Welded Truss Structures in Space", ASME Design Engineering Division, Flexible Assembly Systems, Vol. 48, 1992, pp. 119-123.

9 Dick, Raymond; Terry Faddis and Bill Bar. 'Erectab le truss hardware for large space structures", Proceedings of the $4^{\text {th }}$ International Conference on Engineering, Construction, and Operations In-Space, New York, N.Y., 
1994, pp. 414-22.

10 Brewer, W. V. 'Mechanisms to Implement High Performance Storage and Retrieval of Cylindrical Components for Robotic Assembly of Large Structures in Space", $23^{\text {rd }}$ Biennial Mechanisms Conference, ASME, Vol. 72 part 3, 1994, New York, NY, pp 91-8.

11 Stieber, M.F.; Trudel, C.P. and D.G. Hunter. "Robotic systems for the International Space Station", Proceedings of 1997 IEEE International Conference on Robotics and Automation. Vol: 4, 1997, pp. $3068-73$.

12 Matsueda, T.; Kuraoka, K.; Goma, K.; Sumi, T. and R. Okamura. "JEMRMS system design and development status" Proceedings of 1991 Telesystems Conference. Vol.1., 1991, pp. $391-5$.

13 Heard, Walter L. and Judith J. Watson. 'Results of the ACCESS Space Construction Shuttle Flight Experiment", AIAA Paper Number 86-1186-CP.

14 Hankins, Walter W.; Mixon, Randolph W. and Howard C. Jones. 'Space Truss Assembly Using Teleoperated Manipulators", 1987 Space Applications of Artificial Intelligence and Robotics conference, Greenbelt, Maryland, May 13-14, 1987.

15 Lane, J.C.; Carignan, C. and D. L. Akin. 'Reconfigurable control station design for robotic operations", 1997 IEEE International Conference on Systems, Man, and Cybernetics, Vol. 4 , 1997, pp. 3722 -7.

16 Hirzinger, Gerd; Brunner, Bernhard; Dietrich, Johannes and Johann Heindl. 'Sensor-Based Space Robotics ROTEX and Its Telerobotic Features", IEEE Transaction on Robotics and Automation, Vol. 9, No. 5, Oct. 1993, pp 64963.

17 Oda, Mitsushige and Toshitsugu Doi. 'Teleoperation System of ETS-VII Robot Experiment Satellite", Intelligent Robots and Systems, IROS ' 97, Proceedingsof the 1997 IEEE/RSJ International Conference, Vol. 3, 1997 pp. 1644 50.

18 Oda, M.; Doi, T. and K. Wakata. "Tele-manipulation of a satellite mounted robot by an on-ground astronaut", Proceedings 2001 IEEE International Conference on Robotics and Automation, Vol: 2, pp. $1891-6$.

19 Yoon, Woo-Keun; Goshozono, T.; Kawabe, H.; Kinami, M.; Tsumaki, Y.; Uchiyama, M.; Oda, M. and T. Doi. "Model-based teleoperation of a Space robot on ETS-VII using a haptic interface", Proceedings 2001 IEEE International Conference on Robotics and Automation, Vol. 1 , pp $407-12$.

20 Whittaker, William; Urmson, Chris; Staritz, Peter;Kennedy, Brett and Rob Ambrose. 'Robotics for Assembly, Inspection, and Maintenance of Space Macrofacilities", AIAA paper number 2000-5288.

21 Staritz, P.J.; Skaff, S.; Urmson, C. and W. Whittaker. "Skyworker: a robot for assembly, inspection and maintenance of large scale orbital facilities", Proceedings 2001 IEEE International Conference on Robotics and Automation, Vol: 4 , 2001 pp. 4180 -5.

22 Sydow, P. Daniel; and Cooper, Eric G. Development of a Machine Vision System for Automated Structural Assembly. NASA TM-4366, 1992.

23 William R. Doggett, A Guidance Scheme for Automated Tetrahedral Truss Structure Assembly Based on Machine Vision, NASA TP-3601, November 1996.

24 Ohlund, Kent. 'Path Planner and Real Time System Development Final Report", Mission, Systems and Operations Analysis Task 12, Jan. 1994.

25 Cheryl L. Allen, An Expert System Executive for Automated Assembly of Large Space Truss Structures, NASA TM-4487, November 1993.

26 Doggett, William R.; Rhodes, Marvin D.; Wise, Marion A.; and Armistead, M. F.: "A Smart End-Effector for Assembly of Space Truss Structures." Presented at the Space Operations, Applications, and Research Symposium, July 9-11, 1991, Houston, Texas.

27 Stockman, H. S. (editor) The Next Generation Space Telescope, Visiting a Time When Galaxies Were Young. The Association of Universities for Research in Astronomy, Inc., 1997. 


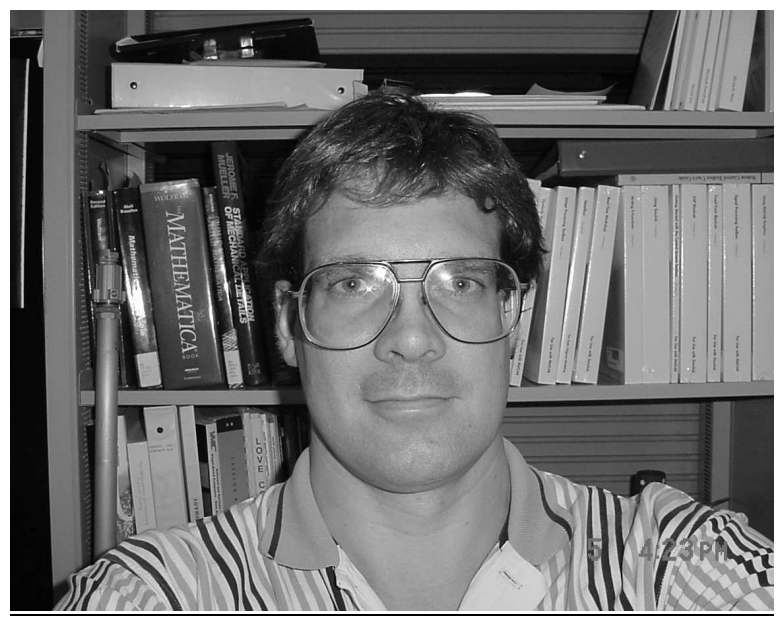

Dr. Doggett is a research engineer in the Systems Integration Branch (SIB) at NASA Langley Research Center. He has a $\mathrm{PhD}$ in Mechanical Engineering and a Master of Science in Electrical Engineering. He has been employed at NASA LaRC since 1987. His primary field of research has been global optimization of robotic paths during maneuvers around obstacles. In the early 90's he lead software and control development for the special purpose end-effectors used to construct a regular tetrahedral truss structure. He also participated in the development of an off-line pathplanning tool to develop robot paths used in the truss construction. Currently Dr. Doggett is involved in the integration of human and robotic operations for in-space assembly operations. 